\title{
Small stakes risk aversion in the laboratory: A reconsideration
}

\author{
Glenn W. Harrison ${ }^{\mathrm{a}, \mathrm{b}, *}$, Morten I. Lau ${ }^{\mathrm{c}, \mathrm{g}}$, Don Ross ${ }^{\mathrm{d}, \mathrm{b}, \mathrm{e}}$, J. Todd Swarthout ${ }^{\mathrm{f}}$ \\ a Department of Risk Management E Insurance and Center for the Economic Analysis of Risk, Robinson College of Business, Georgia State University, USA \\ b School of Economics, University of Cape Town, South Africa \\ c Copenhagen Business School, Denmark \\ d School of Sociology and Philosophy, University College Cork, Ireland \\ e Center for Economic Analysis of Risk, Robinson College of Business, Georgia State University, USA \\ ${ }^{f}$ Department of Economics and Experimental Economics Center, Andrew Young School of Policy Studies, Georgia State University, USA \\ g Durham University Business School, Durham University, England
}

\section{H I G H L I G H T S}

- Prior laboratory experiments provide evidence of low stakes risk aversion (RA).

- Certain assumptions applied to the data imply implausible levels of high stakes RA.

- We conduct an experiment to directly test one of these assumptions.

- The assumption is rejected for a large sample from a population of college students.

- Implausible predictions of large stakes RA do not hold for this population.

\section{A R T I C L E I N F O}

\section{Article history:}

Received 14 June 2017

Received in revised form 22 July 2017

Accepted 4 August 2017

Available online 19 August 2017

\section{JEL classification:}

D81

D03

C91

Keywords:

Risk aversion

Calibration puzzle

Laboratory experiments

\begin{abstract}
A B S T R A C T
Evidence of risk aversion in laboratory settings over small stakes leads to a priori implausible levels of risk aversion over large stakes under certain assumptions. One core assumption in statements of this calibration puzzle is that small-stakes risk aversion is observed over all levels of wealth, or over a "sufficiently large" range of wealth. Although this assumption is viewed as self-evident from the vast experimental literature showing risk aversion over laboratory stakes, it actually requires that lab wealth be varied for a given subject as one evaluates the risk attitudes of the subject. We consider evidence from a simple design that tests this assumption, and find that the assumption is strikingly rejected for a large sample of subjects from a population of college students. We conclude that the implausible predictions that flow from these assumptions do not apply to one specialized population widely used to study economic behavior in laboratory experiments.
\end{abstract}

(c) 2017 Elsevier B.V. All rights reserved.

\section{Introduction}

Debate surrounding theories of decisions under risk and uncertainty has renewed interest in the arguments of the utility function over event outcomes. The local measure of risk aversion proposed by Arrow (1971) and Pratt (1964) for expected utility theory (EUT) is based on terminal wealth being the argument. However, there is nothing in the axiomatic foundation of EUT that requires one to use terminal wealth as the argument: Vickrey (1945) used income instead of terminal wealth; von Neumann and Morganstern

\footnotetext{
* Corresponding author at: Department of Risk Management \& Insurance and Center for the Economic Analysis of Risk, Robinson College of Business, Georgia State University, USA.

E-mail addresses: gharrison@gsu.edu (G.W. Harrison), mla.eco@cbs.dk (M.I. Lau),don.ross@uct.ac.za (D. Ross), swarthout@gsu.edu (J.T. Swarthout).
}

(1953, p. 15-31) were agnostic; and Luce and Raiffa (1957, ch.2) discussed alternatives such as scalar amounts of terminal wealth or income or, alternatively, vectors of commodities. Arrow (1964), Debreu (1959, ch.7) and Hirshleifer (1966) developed models in which the arguments of utility functions are vectors of contingent commodities.

The choice of arguments of the utility function can have important consequences for the inferences one can plausibly draw from empirical estimates of risk attitudes. Many economics experiments present participants with gambles over relatively small stakes and find that such gambles are frequently turned down in favor of less risky gambles with smaller expected values. If the argument of the utility function is terminal wealth, then some patterns of small stakes risk aversion have implausible implications for preferences over gambles where the stakes are no longer small. One example 
from Rabin (2000) is that the expected utility of terminal wealth model implies that an agent who turns down a 50/50 bet of losing $\$ 100$ or gaining $\$ 110$, at all initial wealth levels between $\$ 100$ and $\$ 300,000$, will also, at initial wealth of $\$ 290,000$, turn down a 50/50 bet with possible loss of $\$ 2000$ even when the gain is as large as $\$ 12$ million. Although primarily used as an argument against EUT, it is now well-known that this logic applies to a much wider range of models that assume the argument of the utility function to be terminal wealth (Cox and Sadiraj, 2006; Safra and Segal, 2008). Hence the methodological implications run much deeper than whether EUT is a useful descriptive model of behavior.

Given the importance of understanding the arguments of the utility function, the absence of empirical tests is remarkable. We focus squarely on the specific and influential claim that evidence of small stakes risk aversion in the laboratory generates implausible risk aversion implications for any model in which terminal wealth is the argument of the utility function. ${ }^{1}$ We refer to this claim as the HRC, for "Hansson-Rabin calibration," acknowledging Hansson (1988) and Rabin (2000). We build on a simple theoretical test and experimental design originally developed by Cox and Sadiraj (2008), and independently later by Wilcox (2013); our design follows theirs. Although this design has wider implications, we focus on implications for calibration puzzles defined over terminal wealth models, which are the models that initiated the modern debate.

We present direct evidence that the empirical premiss underlying the HRC claim is false for the typical subjects of laboratory experiments: students in a first-world university. ${ }^{2}$ These subjects exhibit risk aversion for small stakes lotteries for the initial terminal wealth that they bring to the lab, but as we increase the terminal wealth of the subjects they exhibit risk neutrality. We make no claim that this finding generalizes to other populations, fully expect that it could vary from population to population, and encourage tests with different populations. ${ }^{3}$

We review the theoretical statement of the usual calibration puzzle in Section 2, using the simple example from Hansson (1988) since it is not widely known and illustrates the basic points. The generalization by Rabin (2000) can then be quickly stated. Our experimental design is presented in Section 3, and follows Cox and Sadiraj (2008). We evaluate the resulting empirical evidence from 28 binary choices times 590 student subjects in Section 4.

\section{Theory}

What if an individual always rejects a 50:50 lottery offering $x$ and $x+\$ 3$ in favor of $x+\$ 1$ for certain? Without loss of essential generality, assume indifference, so that $u(x+1)=\frac{1}{2} u(x)+\frac{1}{2} u(x+$ 3 ). One solution to this equation is the utility function $u(x)=1-a^{x}$ for $\mathrm{a} \approx 0.618$. It is useful in the sequel to note that this is a bounded function, since $u(x) \rightarrow 1$ as $x \rightarrow \infty$. Now consider increments in utility from $x$ :

$$
\begin{aligned}
& u(x+1)-u(x) \approx 0.382 a^{x} \\
& u(x+\infty)-u(x)=1-\left(1-a^{x}\right)=a^{x} .
\end{aligned}
$$

If (1) and (2) are true, then we can construct "trick lotteries" that make this decision maker look silly. For instance, the decision

\footnotetext{
1 Neilson (2001) and Safra and Segal (2008) also provide concavity calibration claims for terminal wealth models.

2 There have been comparable tests of the premisses of the calibration claims by Cox et al. (2013). One of their experiments involved subjects in Calcutta, India; another involved a casino in Europe and some experimental procedures that are non-standard (in effect, the lab wealth was extremely risky wealth, and plausibly hypothetical wealth from the ex ante perspective of the subject). Wilcox (2013) independently derived similar tests in the laboratory.

3 We now incorporate these simple lottery choices in most batteries we use in the lab and the field for new populations.
}

maker must prefer a certain gain of 1 to a $0<p<0.382$ chance of an arbitrarily large gain $X \gg x$. For instance, $p=1 / 4$, since $1 / 4 a^{x}<0.382 a^{x}$, and $X=\$ 1$ million.

More general conditions for this implausible prediction are now established. If the utility function is bounded on $(0, \infty)$ then that is a sufficient condition for implausible risk aversion in large stakes (e.g., Cox and Sadiraj, 2008; Proposition 2, p.20). Indeed, the only empirical example offered by Rabin (2000) uses a bounded CARA function. On the other hand, small-stakes risk aversion over a large enough finite interval is a sufficient condition for implausible risk aversion for large stakes, whether or not the utility function is bounded.

The HRC puzzle may be stated in terms of four propositions:

- $\mathrm{P} \models$ "the agent is a risk averse EUT maximizer"

- $\mathrm{Q} \models$ "EUT implies full asset integration"

- $\mathrm{R} \models$ "the agent turns down small-stakes gambles in favor of a certain amount with a slightly lower expected value, and does so over a large enough ${ }^{4}$ range of wealth levels W"

- $\mathrm{S} \models$ "the agent turns down large-stakes gambles in favor of a certain amount with a significantly lower value, and looks silly".

The calibration puzzle is the claim that if $\mathrm{P}, \mathrm{Q}$ and $\mathrm{R}$ are true, then $\mathrm{S}$ follows. Since the behavior implied by proposition $\mathrm{S}$ is $a$ priori implausible from a thought experiment, there must be some inconsistency among these propositions. Rabin (2000) draws the implication that P must then be false, and that one should employ models of decision-making under risk that relax proposition $\mathrm{Q}$ such as Cumulative Prospect Theory. As a purely logical matter, of course, this is just one way to resolve this calibration puzzle.

\section{Experimental design}

All of the evidence claimed to support the premiss that decision makers in experiments exhibit small stakes risk aversion for a large enough finite interval comes from designs in which subjects come to the lab with varying levels of wealth and are faced with small-stakes lotteries. This is actually indirect evidence, even if it might be suggestive, since we do not know that different decisionmakers have varying levels of wealth, and there is nothing in EUT that would lead one to assume that they have the same utility function. What is needed is an experimental design that varies the wealth of a given decision-maker, who can be presumed to behave consistently with one utility function during the lab session. ${ }^{5}$

Cox and Sadiraj (2008, p.33) propose an elegant design to test this claim correctly. Give the agent a choice between a safe lottery of $w$ for sure, and a risky lottery of a 50:50 chance of $w-x$ or $w+y$, where $w-x \geq 0$ and $y>x>0$. The key idea is to vary $w$ in the lab. ${ }^{6}$ Consider values of $w$ that can be denoted

\footnotetext{
4 The expression "large enough" is deliberately vague, since it depends on the degree of risk aversion exhibited under proposition $\mathrm{P}$, and the lotteries in proposition $\mathrm{S}$ that a priori seem to generate silly behavior.

5 Thought experiments along these lines were developed by Watt (2002) and Palacios-Huerta and Serrano (2006), and showed that the implied risk aversion underlying proposition $\mathrm{R}$ are a priori implausible. Despite minor errors in some of the calculations of the latter, noted at http://www.econ.brown.edu/Faculty/ serrano/disclaimers/2006EL91dis.html, the message does not change.

6 Cox and Sadiraj (2008, p.32) explain why one cannot test proposition R by only asking one lottery choice question of this kind at only one level of lab wealth, as in Barberis et al. (2006, p. 1071) and Schechter (2007). In response to Watt (2002), Rabin and Thaler (2002, p.230) make exactly this mistake in misunderstanding the existing experimental literature: "We refer any reader who believes in risk neutrality to pick up virtually any experimental test of risk attitudes. Dozens of laboratory experiments show that people are averse to far more favorable bets for smaller stakes. The idea that people are not risk neutral in playing for modest stakes is uncontroversial; indeed, nobody to our knowledge interprets the existing evidence as arguing that expected-value maximization (risk neutrality) is a good fit". The tests proposed here address the hypothesis of expected-value maximization for the relevant theoretical proposition $\mathrm{R}$.
} 
$\mathrm{w}, \mathrm{w}, \mathrm{w}, \mathrm{w}, \mathrm{w}, \mathrm{W}, \mathrm{W}$, where smaller values of the letter $w$ denote smaller values of lab wealth. Consider values like these for lab wealth that may be plausibly much less than the $\mathbf{W}$ that the subject has in the field prior to the experiment. The experimenter does not need to know $\mathbf{W}$ for a given subject, but by varying "lab wealth" for that subject the experimenter has considered small-stakes lottery choices over a loss of $x$ and a gain of $y$ for "field + lab" wealth levels $\mathbf{W}+{ }_{\mathrm{w}}, \mathbf{W}+_{\mathrm{w}}, \mathbf{W}+_{\mathrm{w}}, \mathbf{W}+_{\mathrm{w}}, \mathbf{W}+\mathrm{w}, \mathbf{W}+\mathrm{w}$ and $\mathbf{W}+\mathbf{W}$, respectively, for that subject. This step of the design presumes that we vary lab wealth for a given subject, since then we can presume that field wealth $\mathbf{W}$ is constant for the experimental session. ${ }^{7}$ This step of the experimental design also assumes proposition $\mathrm{Q}$, that the agent perfectly asset integrates field wealth and lab wealth.

If the agent prefers the safe lottery over the risky lottery for all of the lab wealth values the experimenter's budget can afford, then we have verification of proposition $R$, at least for the range of "field + lab" wealth proscribed by the experimenter's budget. If we observe the agent choosing the safe lottery for small levels of lab wealth but the risky lottery for larger levels of lab wealth, then proposition $\mathrm{R}$ is rejected for that agent. Of course, we do not expect deterministic patterns of choice, so one ought to make some claim about the statistical significance of these choice patterns. This is why we have 28 choices for each subject. Another nice feature of this experimental design is that we need not structurally model the EUT decision process for the agent: we can rely on simple statistical models such as (panel) probit, conditioned on lab wealth.

Table 1 shows the 28 lottery pairs we used. The smallest lab wealth was $\$ 15$ and the largest lab wealth was $\$ 135$. Table 1 shows that 20 of the choices involve 50:50 risky lotteries, and 8 of the choices involve 90:10 risky lotteries to allow relatively large outcomes for the risky lottery. The order of lotteries in Table 1 is solely for pedagogic convenience: the order of presentation was randomized independently for each subject. One lottery is the safe lottery, and the other lottery is the risky lottery. The far right columns of Table 1 show the Expected Value (EV) of each choice, and the difference in favor of the risky lottery. Since the risky lottery provided an EV that always slightly exceeded the EV of the safe lottery, we know that a choice of the safe lottery implies risk aversion under EUT. Since the EV difference is strictly positive, choices of the risky lottery are consistent with risk neutrality or risk preference under EUT.

The first 14 lottery choices reflect lower levels of lab wealth between $\$ 15$ and $\$ 30$, and the last 14 lottery choices, shaded in Table 1, reflect higher levels of lab wealth between $\$ 120$ and $\$ 135$. There are repetitions of the $-x$ and $+y$ values at different levels of lab wealth: hence the design builds in controls for the same losses and gains relative to lab wealth, and only varies lab wealth. For instance, choice pairs 2 and 26 have $x=8$ and $y=10$, with lab wealth of $\$ 15$ and $\$ 135$, respectively. So apart from the change in lab wealth, these are the same risky lotteries, as required by proposition R. Similarly, choice pairs 9 and 18 have $x=18$ and $y=21$ with lab wealth of $\$ 25$ and $\$ 125$, respectively. There is variation in the values of $x$ and $y$, and the difference between $x$ and $y$ : the smallest difference between $x$ and $y$ is $\$ 1$ (e.g., choice pairs $3,6,7$ ) and the largest difference is $\$ 85$ (choice pairs $1,12,22$ and 27).

\section{Evidence}

The battery in Table 1 was administered, in random order, to 590 undergraduates recruited from the Georgia State University undergraduate population. This battery was administered as part of a larger experiment, in which subjects made a total of 100 binary

\footnotetext{
7 In other words, one cannot implement this aspect of the design on a betweensubjects basis, or the theoretical premiss could be violated by heterogeneity.
}

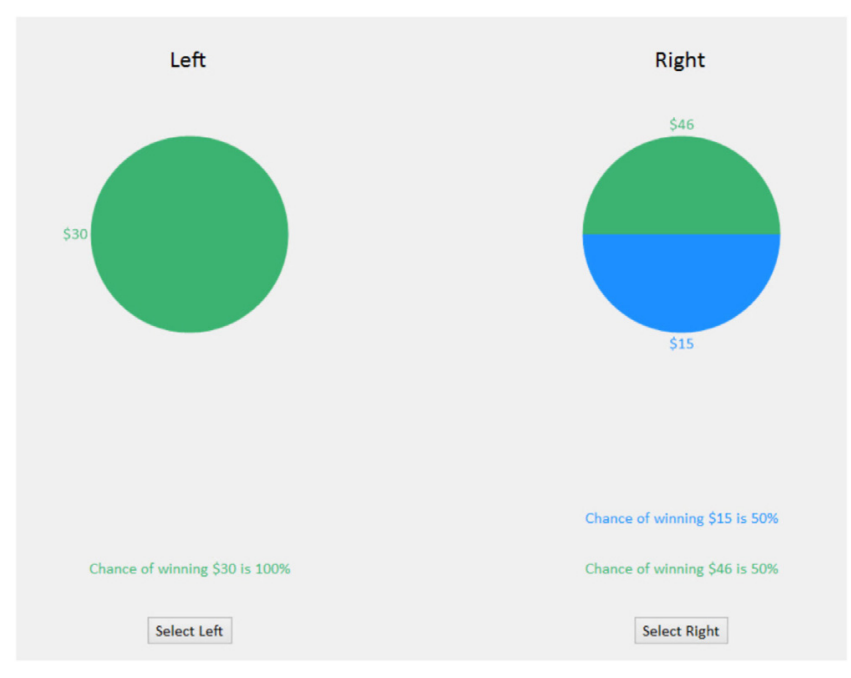

Fig. 1. Binary choice display.

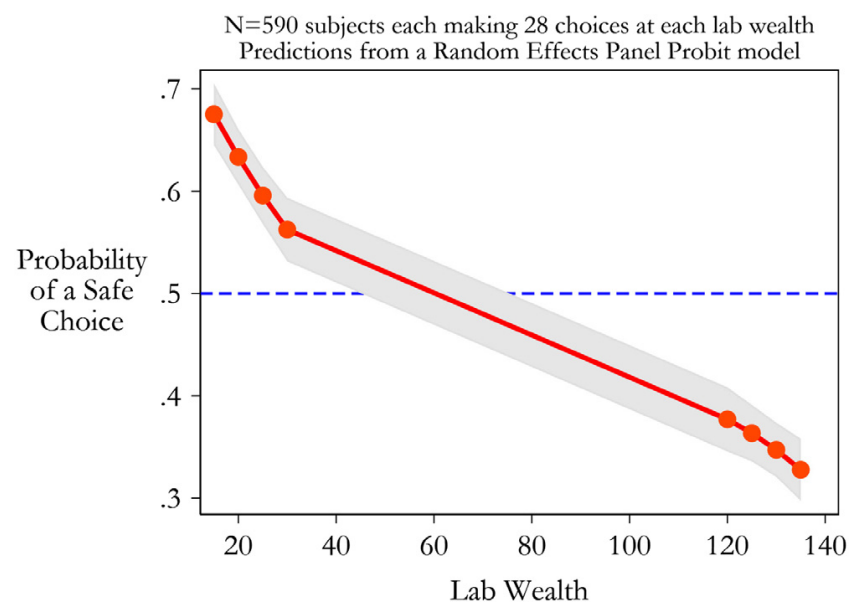

Fig. 2. Predicted probability of a risk averse choice at varying levels of lab wealth.

choices, spanning gain, loss and mixed frames. A typical display is shown in Fig. 1. ${ }^{8}$ Indifference was not permitted as an option. Subjects also completed a demographics survey, and subsequently participated in a "lab casino" in which choices mimicked those found in video slot machines. Each subject received a \$5 showup payment. One feature of this experiment is that participation times in a given session were staggered, so that several subjects joined the session at 30-minute intervals. All instructions were then administered in written form, and with a video presentation on each computer that the subject listened to privately with headphones. This procedure was used to avoid subjects feeling any peer pressure to finish early or late. At the end of the 100 lottery choices, one choice was selected at random with physical dice, and then the lottery chosen was played out with physical dice. ${ }^{9}$ Subjects were

\footnotetext{
8 These lottery choices were embedded in a larger battery that included some choices in which there were losses out of house endowments displayed for those choices. When there were no losses, the display did not mention any house endowment, as in Fig. 1.

9 We opted for using the Random Lottery Incentive Method (RLIM), where one of the 100 choices was chosen at random for playing out and payment. We did so for two reasons, recognizing that this is not as innocent a procedure as some maintain (see Harrison and Swarthout, 2014; Cox et al., 2015 and Brown and Healy, 2016 for evidence and extensive literature discussion). The first reason was to ensure that
} 
Table 1

Experimental design.

All currency values in U.S. Dollars

\begin{tabular}{|c|c|c|c|c|c|c|c|c|c|c|}
\hline \multirow[b]{2}{*}{ ID \# } & \multirow{2}{*}{$\begin{array}{c}\text { Lab } \\
\text { Wealth }\end{array}$} & \multicolumn{4}{|c|}{ Risky Lottery } & \multirow[b]{2}{*}{$-\mathrm{x}$} & \multirow[b]{2}{*}{$\mathrm{y}$} & \multicolumn{3}{|c|}{ Expected Value } \\
\hline & & Prob $^{\text {low }}$ & Payoff ${ }^{\text {low }}$ & Prob $^{\text {hi }}$ & Payoff $^{\text {hi }}$ & & & Safe & Risky & Difference \\
\hline 1 & 15 & 0.9 & 5 & 0.1 & 110 & -10 & 95 & 15 & 15.5 & 0.5 \\
\hline 2 & 15 & 0.5 & 7 & 0.5 & 25 & -8 & 10 & 15 & 16 & 1 \\
\hline 3 & 15 & 0.5 & 7 & 0.5 & 24 & -8 & 9 & 15 & 15.5 & 0.5 \\
\hline 4 & 20 & 0.5 & 10 & 0.5 & 32 & -10 & 12 & 20 & 21 & 1 \\
\hline 5 & 20 & 0.9 & 15 & 0.1 & 70 & -5 & 50 & 20 & 20.5 & 0.5 \\
\hline 6 & 20 & 0.5 & 10 & 0.5 & 31 & -10 & 11 & 20 & 20.5 & 0.5 \\
\hline 7 & 20 & 0.5 & 2 & 0.5 & 39 & -18 & 19 & 20 & 20.5 & 0.5 \\
\hline 8 & 25 & 0.5 & 12 & 0.5 & 40 & -13 & 15 & 25 & 26 & 1 \\
\hline 9 & 25 & 0.5 & 7 & 0.5 & 46 & -18 & 21 & 25 & 26.5 & 1.5 \\
\hline 10 & 25 & 0.5 & 10 & 0.5 & 43 & -15 & 18 & 25 & 26.5 & 1.5 \\
\hline 11 & 30 & 0.9 & 25 & 0.1 & 90 & -5 & 60 & 30 & 31.5 & 1.5 \\
\hline 12 & 30 & 0.9 & 20 & 0.1 & 125 & -10 & 95 & 30 & 30.5 & 0.5 \\
\hline 13 & 30 & 0.5 & 18 & 0.5 & 45 & -12 & 15 & 30 & 31.5 & 1.5 \\
\hline 14 & 30 & 0.5 & 15 & 0.5 & 46 & -15 & 16 & 30 & 30.5 & 0.5 \\
\hline 15 & 120 & 0.5 & 108 & 0.5 & 135 & -12 & 15 & 120 & 121.5 & 1.5 \\
\hline 16 & 120 & 0.5 & 110 & 0.5 & 132 & -10 & 12 & 120 & 121 & 1 \\
\hline 17 & 120 & 0.9 & 115 & 0.1 & 180 & -5 & 60 & 120 & 121.5 & 1.5 \\
\hline 18 & 125 & 0.5 & 107 & 0.5 & 146 & -18 & 21 & 125 & 126.5 & 1.5 \\
\hline 19 & 125 & 0.5 & 110 & 0.5 & 143 & -15 & 18 & 125 & 126.5 & 1.5 \\
\hline 20 & 125 & 0.5 & 112 & 0.5 & 140 & -13 & 15 & 125 & 126 & 1 \\
\hline 21 & 130 & 0.9 & 125 & 0.1 & 180 & -5 & 50 & 130 & 130.5 & 0.5 \\
\hline 22 & 130 & 0.9 & 120 & 0.1 & 225 & -10 & 95 & 130 & 130.5 & 0.5 \\
\hline 23 & 130 & 0.5 & 112 & 0.5 & 149 & -18 & 19 & 130 & 130.5 & 0.5 \\
\hline 24 & 130 & 0.5 & 120 & 0.5 & 141 & -10 & 11 & 130 & 130.5 & 0.5 \\
\hline 25 & 130 & 0.5 & 115 & 0.5 & 146 & -15 & 16 & 130 & 130.5 & 0.5 \\
\hline 26 & 135 & 0.5 & 127 & 0.5 & 145 & -8 & 10 & 135 & 136 & 1 \\
\hline 27 & 135 & 0.9 & 125 & 0.1 & 230 & -10 & 95 & 135 & 135.5 & 0.5 \\
\hline 28 & 135 & 0.5 & 127 & 0.5 & 144 & -8 & 9 & 135 & 135.5 & 0.5 \\
\hline
\end{tabular}

Table 2

Predicted safe choices from alternative statistical models.

\begin{tabular}{|c|c|c|c|c|c|c|c|c|c|}
\hline \multirow{3}{*}{$\begin{array}{c}\text { Lab } \\
\text { Wealth } \\
\$ 15\end{array}$} & \multicolumn{3}{|c|}{ Probit } & \multicolumn{3}{|c|}{ Random Effects Probit } & \multicolumn{3}{|c|}{ Semi-Non-Parametric (SNP) } \\
\hline & \multirow{2}{*}{$\frac{\text { Mean }}{0.64}$} & \multicolumn{2}{|c|}{$95 \% \mathrm{CI}$} & \multirow{2}{*}{$\frac{\text { Mean }}{0.68}$} & \multicolumn{2}{|c|}{$95 \% \mathrm{CI}$} & \multirow{2}{*}{$\frac{\text { Mean }}{0.64}$} & \multicolumn{2}{|c|}{$95 \% \mathrm{CI}$} \\
\hline & & 0.62 & 0.67 & & 0.65 & 0.71 & & 0.62 & 0.66 \\
\hline$\$ 20$ & 0.61 & 0.59 & 0.63 & 0.63 & 0.61 & 0.66 & 0.61 & 0.60 & 0.62 \\
\hline$\$ 25$ & 0.58 & 0.55 & 0.60 & 0.60 & 0.57 & 0.62 & 0.58 & 0.57 & 0.59 \\
\hline$\$ 30$ & 0.55 & 0.53 & 0.57 & 0.56 & 0.53 & 0.59 & 0.55 & 0.53 & 0.56 \\
\hline$-\overline{\$ 120}$ & $\overline{0.40}$ & $\overline{0.37}$ & - $0 . \overline{43}$ & $\overline{0.38}$ & - 0.35 & - - & $\overline{0.40}$ & - & - \\
\hline$\$ 125$ & 0.39 & 0.36 & 0.41 & 0.36 & 0.34 & 0.39 & 0.39 & 0.38 & 0.40 \\
\hline$\$ 130$ & 0.38 & 0.35 & 0.40 & 0.35 & 0.32 & 0.37 & 0.38 & 0.36 & 0.39 \\
\hline$\$ 135$ & 0.36 & 0.33 & 0.39 & 0.33 & 0.30 & 0.36 & 0.36 & 0.34 & 0.38 \\
\hline
\end{tabular}

paid privately. The instructions are reproduced in an Appendix A (available on request).

Fig. 2 displays the basic results, using a simple (random effects, panel) probit specification in which the binary choice is the dependent variable and the explanatory variable is lab wealth. For the probit specifications we use quadratic and cubic terms

we collected choices over a wide enough array of lotteries to be able to test the premiss using within-subject data. If we had opted for giving one choice to each subject, to avoid using the RLIM, this would have been infeasible and would have required an extreme assumption of preference homogeneity across subjects. The second reason was that the null hypothesis being tested is normally stated assuming EUT, and RLIM is valid under EUT. to capture possible non-linearity in the estimated latent index. Standard errors allow for clustering by subject. The solid line shows the average prediction, and the shaded area shows the $95 \%$ confidence interval around that prediction. The results are clear: subjects exhibit some risk aversion at low levels of lab wealth and exhibit risk neutrality (or risk preference) at higher levels of lab wealth. Although relying on interpolation, the switch point appears to be around a lab wealth of $\$ 60$. By this evidence we infer that proposition $\mathrm{R}$ is false for this population and these lottery choices. ${ }^{10}$

10 The qualitative results are even stronger if one drops the "skewed" risky lotteries, which are those numbered 1, 5, 11, 12, 17, 21, 22 and 27 in Table 1. 
Table 2 lists estimates of the probability of safe choices using several alternative statistical specifications. One specification is a standard probit model, and another specification is a random effects probit model; both use quadratic and cubic terms in lab wealth. A third specification is the semi-nonparametric (SNP) approach of Gallant and Nychka (1987). Although less parametric than the probit specifications, the SNP estimates do not account for the panel nature of these data. Our preferred specification is the random effects, panel probit, shaded in Table 2. The same qualitative conclusion applies for all three specifications. The random effects probit implies more extreme predictions above and below $1 / 2$ than the other two specifications, but in all cases the $95 \%$ confidence intervals lie above $1 / 2$ for small levels of lab wealth, and lie below $1 / 2$ for higher levels of lab wealth.

\section{Conclusions}

For a sample from a population that is similar to populations widely used to study behavior in experiments, the empirical basis for the calibration puzzle does not exist.

\section{Acknowledgments}

We are grateful to Jim Cox, Elisabet Rutström, Vjollca Sadiraj and Nathaniel Wilcox for valuable comments and discussion. Financial support was provided by the Danish Social Science Research Council (Project \#12-130950).

\section{Appendix A. Supplementary data}

Supplementary material related to this article can be found online at http://dx.doi.org/10.1016/j.econlet.2017.08.003.

\section{References}

Arrow, K.J., 1964. The role of securities in the optimal allocation of risk-bearing. Rev. Econom. Stud. 31, 91-96.

Arrow, K.J., 1971. Essays in the Theory of Risk-Bearing. Markham, Chicago.

Barberis, N., Huang, M., Thaler, R.H., 2006. Individual preferences, monetary gambles, and stock market participation: A case for narrow framing. Amer. Econ. Rev. 96 (4), 1069-1090.
Brown, A.L., Healy, P.J., 2016. Separated Decisions. Working Paper \#16-02. Department of Economics, Ohio State University.

Cox, J.C., Sadiraj, V., 2006. Small- and large-stakes risk aversion: Implications of concavity calibration for decision theory. Games Econom. Behav. 56, 45-60.

Cox, J.C., Sadiraj, V., 2008. Risky decisions in the large and in the small: Theory and experiment. In: Cox, J.C., Harrison, G.W. (Eds.), Risk Aversion in Experiments. In: Research in Experimental Economics, vol. 12, Emerald, Bingley UK.

Cox, J.C., Sadiraj, V., Schmidt, U., 2015. Paradoxes and mechanisms for choice under risk. Exp. Econ. 18, 215-250.

Cox, J.C., Sadiraj, V., Vogt, B., Dasgupta, U., 2013. Is there a plausible theory for decision under risk? A dual calibration critique. Econom. Theory 54 (2), 305333.

Debreu, G., 1959. Theory of Value. John Wiley and Sons, New York.

Gallant, A.R., Nychka, D.W., 1987. Semi-nonparametric maximum likelihood estimation. Econometrica 55, 363-390.

Hansson, B., 1988. Risk aversion as a problem of conjoint measurement. In: Gardenfors, P., Sahlin, N.-E. (Eds.), Decisions, Probability, and Utility. Cambridge University Press, New York.

Harrison, G.W., Swarthout, J.T., 2014. Experimental payment protocols and the bipolar behaviorist. Theory and Decision 77, 423-438.

Hirshleifer, J., 1966. Investment decision under uncertainty: Applications of the state-preference approach. Quart. J. Econ. 80 (2), 252-277.

Luce, R.D., Raiffa, H., 1957. Games and Decisions: Introduction and Critical Survey. Wiley, New York.

Neilson, W.S., 2001. Calibration results for rank-dependent expected utility. Econ. Bull. 4, 1-5.

Palacios-Huerta, I., Serrano, R., 2006. Rejecting small gambles under expected utility. Econom. Lett. 91 (2), 250-259.

Pratt, John W., 1964. Risk aversion in the small and in the large. Econometrica 32, $123-136$.

Rabin, Matthew, 2000. Risk aversion and expected utility theory: A calibration theorem. Econometrica 68, 1281-1292.

Rabin, Matthew, Thaler, Richard H., 2002. Defending expected utility theory: Response. J. Econ. Perspect. 16 (2), 229-230.

Safra, Zvi, Segal, Uzi, 2008. Calibration results for non-expected utility theories. Econometrica 76 (5), 1143-1166.

Schechter, Laura, 2007. Risk aversion and expected-utility theory: A calibration exercise. J. Risk Uncertain. 35, 67-76.

Vickrey, William, 1945. Measuring marginal utility by reactions to risk. Econometrica 13 (4), 319-333.

von Neumann, John, Morganstern, Oskar, 1953. Theory of Games and Economic Behavior, third ed.. Princeton University Press, Princeton.

Watt, Richard, 2002. Defending expected utility theory: Comment. J. Econ. Perspect. 16 (2), 227-229.

Wilcox, Nathaniel T., 2013. Is the Premise of Risk Calibration Theorems Plausible? Presentation, CEAR Workshop, Durham University, September 17, 2013. 